\title{
Anti-oestrogenic Hormone Therapy And Risk Factors For Depressive Disorder In Women With Breast Carcinoma Under Adjuvant Treatment: A Systematic Review Of The Literature
}

\author{
Oliveira C. ${ }^{1}$, Ramos J. ${ }^{1}$, Nascimento M. ${ }^{2}$,Martins-Branco D. ${ }^{3}$, Gouveia E. ${ }^{3}$, Monteiro I. ${ }^{4}$
}

1 - Psychiatry Department, Hospital Professor Doutor Fernando Fonseca

2 - Psychiatry Department, Hospital Vila Franca de Xira

3 - Medical Oncology Department, Instituto Português de Oncologia de Lisboa FG

4 -Psychiatry Department, Instituto Português de Oncologia de Lisboa FG

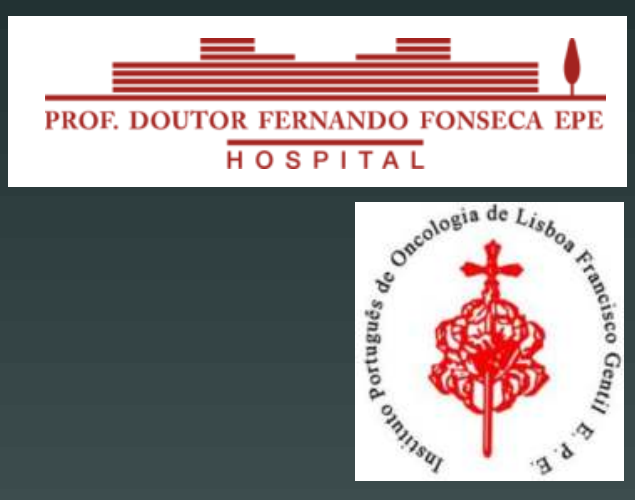

\section{INTRODUCTION AND OBJECTIVES}

Women with breast cancer have an increased risk of depression.

Anti-oestrogenic hormone therapy is often indicated as a possible cause.

We aim to summarize evidence on this topic and to address factors associated with depression in breast cancer survivors under hormone therapy.

Specific goals:
I) validate antiestrogen therapy as an independent factor to increase the risk of developing depressive disorder;
II) look for differences between antiestrogenic hormone therapies in the development of depressive disorder;
III) identify the factors associated with a higher risk of developing depressive disorder under anti-estrogen therapy;
IV) determine the most used scale to diagnose the depressive disorder in women under anti-estrogen therapy.

\section{METHODS}

Systematic review of the literature using 3 electronic databases (Medline, Embase and, PsycINFO) until December 31, 2016. Only randomized controlled trials (RCTs) and observational studies of adult women with non-metastatic breast cancer under adjuvant treatment were included.

\section{RESULTS}

We identified 393 articles, 23 met the inclusion criteria:

- 5 RCTs and 18 observational studies

EMBASE, MEDLINE,

PsycINFO (n=393)

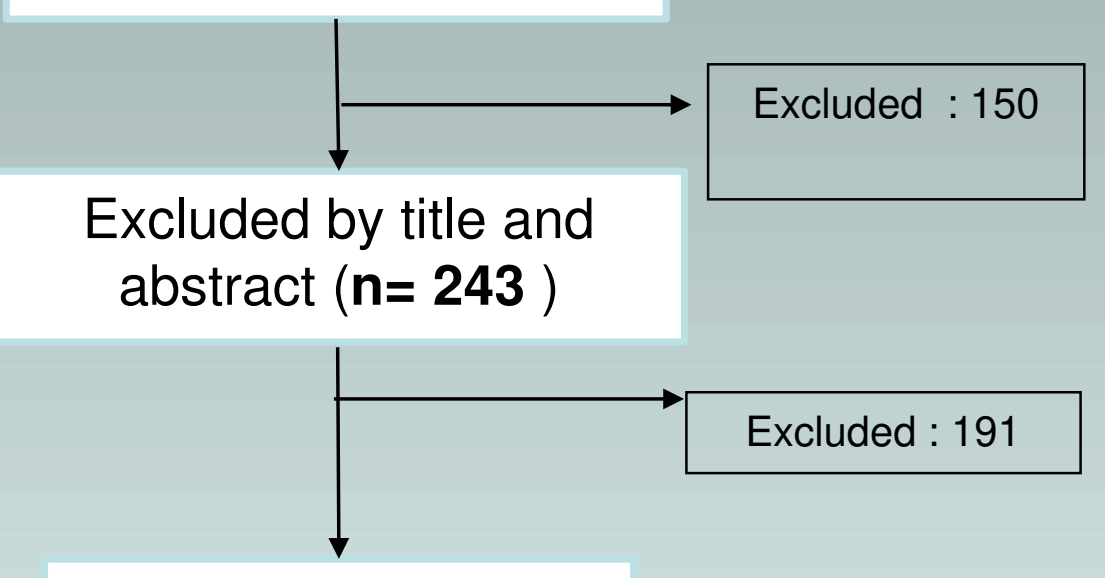

Total accepted after

full review $(\mathbf{n}=\mathbf{5 2})$

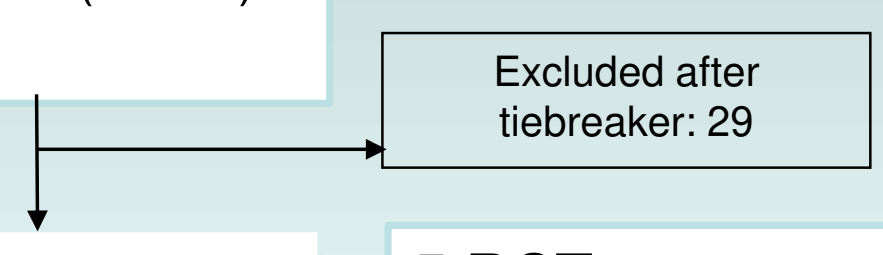

Total included

$(n=23)$
5 RCTs

18 observational studies

\section{Specific Objectives \\ RESULTS SUMMARY}

I 11 observational studies analysed hormone therapy as an independent factor for depression:

- 2 favoured the reduction of risk

- 4 showed association with depression

- 5 showed no association

II 7 studies (4 RCTs and 3 observational studies) reported no differences between hormone therapy drugs

III 12 studies identified factors associated with depression during hormone therapy:

- 1 RCT demonstrated that physical exercise and hypocaloric diet might reduce the risk of depression;

- 11 observational studies identified the following risk factors:

1. active smoking $(n=1)$,

2. psychosocial variables $(n=1)$,

3. menopausal symptoms $(n=2)$, chemotherapy $(n=2)$

4. expression of oestrogen receptors in the tumour $(n=1)$.

IV The most frequently used scale was "Beck Depression Inventory"

\section{CONCLUSIONS}

The role of hormone therapy in the emergence of depression in breast cancer survivors remains unclear. However, the possibility that hormone therapy induces depressive states in a vulnerable subgroup of patients should not be excluded. This review identified risk factors and valid tools for evaluating depression that should be considered in future prospective studies. 